\title{
After insufficient radiofrequency ablation, tumor- associated endothelial cells exhibit enhanced angiogenesis and promote invasiveness of residual hepatocellular carcinoma
}

Jian Kong ${ }^{1}$, Lingqun Kong ${ }^{2}$, Jinge Kong ${ }^{3}$, Shan Ke ${ }^{1}$, Jun Gao ${ }^{1}$, Xuemei Ding ${ }^{1}$, Lemin Zheng ${ }^{3}$, Huichuan Sun ${ }^{2 *}$ and Wenbing Sun ${ }^{1 *}$

\begin{abstract}
Background: The mechanism regarding rapid progression of residual hepatocellular carcinoma (HCC) after insufficient radiofrequency ablation (RFA) has been preliminarily discussed. However, most studies have mainly focused on RFA-induced changes in the tumor cells. The present study was designed to determine whether tumorassociated endothelial cells (TAECS) could contribute to the invasiveness of HCC after insufficient RFA.

Methods: TAECs were isolated from fresh HCC tissue and characterized. Morphological changes were observed in TAECs after heat treatment for 10 min. TAEC proliferation, migration and tube formation after heat treatment for 10 min at $37^{\circ} \mathrm{C}$ (control group), and 42 and $47^{\circ} \mathrm{C}$ (insufficient RFA groups) were examined. The differences in TAECs interactions with HepG2-GFP or HCCLM3-GFP cells among the two insufficient RFA groups and control group were evaluated. The expression of E-selectin, ICAM-1 and VCAM-1 in TAECs was measured. The effects of TAECs on the invasiveness of HepG2-GFP or HCCLM3-GFP cells after insufficient RFA were analyzed. The IL-6, IL-8, MCP-1 and GRO-a concentrations in conditioned medium from TAECs were measured after insufficient RFA. The associated signaling pathways of Akt, ERK1/2, STAT3 and NF-KB were analyzed in TAECs after insufficient RFA.

Results: TAECs expressed the EC-specific markers and took up complexes of Dil-Ac-LDL. Relative to the control group, the proliferation of TAECs was significantly inhibited and their migration and tube formation were significantly enhanced in the insufficient RFA groups. Significantly more HepG2-GFP or HCCLM3-GFP cells adhered to TACEs in these groups than in the control group (all $P<0.001$ ), via up-regulated expression of E-selectin, ICAM-1 and VCAM-1. TAECs promoted the invasiveness of HepG2-GFP or HCCLM3-GFP cells after insufficient RFA via the up-regulation of IL-6, IL-8, MCP-1 and GRO-a in conditioned medium (all $P<0.05$ ). Insufficient RFA enhanced the activities of Akt, ERK1/2 and NF-KB signaling pathways and inhibited STAT3 signaling pathways.

Conclusions: Insufficient RFA enhanced TAEC migration and tube formation, and this may play a key role in the rapid growth of residual HCC. Increased expression of metastasis-related molecules in TAECs after insufficient RFA may be a potential mechanism for the metastasis of residual HCC.
\end{abstract}

Keywords: Radiofrequency ablation, Hepatocellular carcinoma, Tumor-associated endothelial cells, Invasiveness, Metastasis

\footnotetext{
* Correspondence: sun.huichuan@zs-hospital.sh.cn; wb.sun@yahoo.com.cn

${ }^{2}$ Liver Cancer Institute, Zhongshan Hospital, Fudan University, Shanghai,

China

'Department of Hepatobiliary Surgery, Beijing Chaoyang Hospital, Capital

Medical University, Beijing, China

Full list of author information is available at the end of the article
} 


\section{Background}

Hepatocellular carcinoma (HCC) is the sixth most common neoplasm and the third most frequent cause of cancer death [1]. Most HCC patients have underlying cirrhosis, which complicates the management of their cancer, especially in the case of early $\mathrm{HCC}$ in the setting of well-compensated cirrhosis [2]. Recently, radiofrequency ablation (RFA) has been considered as being potentially curative for early-stage $\mathrm{HCC}$ in patients with or without surgical prospects owing to its ease of use, safety and cost-effectiveness, and the fact that it is minimally invasive [3]. However, one of the major problems with RFA is the difficulty in achieving complete tumor destruction [4]. Because of the heat sink effect of blood vessels or the fact that the periphery of the tumor is distant from the center of ablation, the target temperature for ablation can not be easily achieved throughout the tumor; consequently, residual $\mathrm{HCC}$ can be present after RFA [5].

In the past decade, rapid tumor progression and sarcomatous changes after RFA have been reported in increasing numbers of clinical centers [6-8]. In particular, rapid tumor progression after RFA, which may mostly be associated with the progression of residual HCC, has been gaining increasing attention, and several underlying mechanisms for this phenomenon have been proposed. Our previous studies have demonstrated that insufficient RFA could facilitate rapid progression in residual hepatic VX2 carcinoma due to the induction of the overexpression of several molecular factors, and also promoted angiogenesis in residual HCC via hypoxiainducible factor- $1 \alpha$ (HIF-1 $\alpha)$ /vascular endothelial growth factor-A (VEGFA) $[9,10]$. Another study also showed that insufficient RFA therapy may induce further malignant transformation of HCC [11]. However, these studies have mainly been focused on RFA-induced changes to the tumor cells. Whether or not non-tumor cells are also affected by insufficient RFA, and consequently contribute to progression of tumor cells, is still poorly understood.

In primary tumors, cancer cells are surrounded by a complex microenvironment comprised of numerous cells including endothelial cells present in the blood and lymphatic circulation, stromal fibroblasts and a variety of bone marrow-derived cells [12]. Evidence is accumulating that the altered microenvironment after RFA may enhance the outgrowth of residual tumor cells [4,13-15]. Tumor-associated endothelial cells (TAECs), as an important component of the microenvironment of tumor, play a key role in angiogenesis. This is crucial for the growth and invasion of solid tumors, as the vasculature provides metabolic support for tumor cells and serves as the gatekeeper for their escape and entry into the circulation [16]. What is more, evidence also shows that
TAECs express tumor-specific endothelial markers and contain several regulated secretory organelles. These include Weibel-Palade bodies, the tissue plasminogen activator (tPA) organelle, and the type- 2 chemokinecontaining organelle, which are responsible for the secretion of tPA, cytokines IL-8 and IL-6, monocyte chemoattractant protein-1 (MCP-1), and growth-regulated oncogene- $\alpha$ (GRO- $\alpha)$ [17-19]. It has yet to be established if insufficient RFA enhances angiogenesis in TAECs. Whether or not TAECs promote the invasiveness of residual HCC via secreted cytokines and help residual tumor cells intravasate into the circulation after insufficient RFA also requires further investigation.

In the present study, we explored the biological behavioral changes in TAECs after insufficient RFA, and whether or not insufficient RFA promoted the metastasis of hepatoma cells via the enhanced secretion of cytokines and expression of adhesion molecules in TAECs.

\section{Materials and methods Patients}

Fresh HCC tissues were obtained from seven patients who had positive $\alpha$-fetoprotein (AFP) with curative liver resection at the Liver Cancer Institute and Zhongshan Hospital, Fudan University (Shanghai, China). The study design was approved by the Ethics Committee of Fudan University, and all the study participants provided informed consent.

\section{Cell lines and cell culture}

Stable green fluorescent protein (GFP)-expressing HepG2 and HCCLM3 cells, two human HCC cell lines established at the Liver Cancer Institute and Zhongshan Hospital [20,21], were maintained in high-glucose DMEM (Gibco, Los Angeles, USA) supplemented with $10 \%$ fetal bovine serum (FBS; Gibco, Los Angeles, USA), $100 \mathrm{U} / \mathrm{ml}$ penicillin and $100 \mu \mathrm{g} / \mathrm{ml}$ streptomycin in a humidified atmosphere of $5 \% \mathrm{CO}_{2}$ at $37^{\circ} \mathrm{C}$. TAECs were isolated as described before [22]. Briefly, TAECs were obtained from surgical HCC specimens immediately after removal from patients. Specimens were minced and digested by incubation for $1 \mathrm{~h}$ at $37^{\circ} \mathrm{C}$ in 1640 medium (Gibco, Los Angeles, USA) containing 0.1\% collagenase IV (Sigma-Aldrich, St. Louis, MO, USA). After washing in PBS (Gibco, Los Angeles, USA), the cell suspension was forced through a graded series of meshes to separate the cell components from stroma and aggregates. TAECs were isolated from cell suspension using anti-CD31 monoclonal antibodies (mAbs) coupled to magnetic beads (Miltenyi Biotech, Bergisch Gladbach, Germany) and magnetic cell-sorting using the MACS system (Miltenyi Biotech, Bergisch Gladbach, Germany). To increase the purity of isolated TAECs after positive magnetic bead isolation, the cell pellets underwent a 
second isolation with anti-CD31 mAbs. Cells were grown in complete EGM-2 medium (Lonza, Basel, Switzerland) supplemented with $10 \% \mathrm{FBS}, 100 \mathrm{U} / \mathrm{ml}$ penicillin and $100 \mu \mathrm{g} / \mathrm{ml}$ streptomycin in a humidified atmosphere of $5 \% \mathrm{CO}_{2}$ at $37^{\circ} \mathrm{C}$. TAECs were used at passages 1-6.

\section{Immunofluorescence analysis}

TAECs were grown on 24-well plates to $40-50 \%$ confluence, then fixed and blocked. Cells were then incubated with primary monoclonal or polyclonal antibodies against CD31, CD34, vascular endothelial growth factor receptor-1 (VEGFR1), vascular endothelial growth factor receptor-2 (VEGFR2), von Willebrand factor (vWF), CD68 and aSMA (Santa Cruz, California, USA) overnight at $4^{\circ} \mathrm{C}$. The next day, plates were washed and incubated with antimouse or anti-rabbit fluorescein isothiocyanate- and/or tetramethyl rhodamine isothiocyanate-conjugated secondary antibody (Invitrogen, Carlsbad, USA). Cells were counterstained with 4'-6-diamidino-2-phenylindole (DAPI; KeyGen Biotech, Nanjing, China) to visualize cell nuclei and observed using an inverted fluorescence microscopy (Olympus IX51) equipped with an Olympus Qcolor 3 digital camera (Olympus).

\section{Internalization of acetylated low-density lipoprotein}

TAECs were incubated in serum-free EBM-2 medium containing $10 \mu \mathrm{g} / \mathrm{ml}$ rhodamine-labeled 1,1V-dioctadecyl3,3,3V,3V-tetramethylindocarbocyanine acetylated lowdensity lipoprotein (Dil-Ac-LDL; Sigma-Aldrich, St. Louis, $\mathrm{MO}$, USA) for $4 \mathrm{~h}$ at $37^{\circ} \mathrm{C}$. The cells were fixed in 4\% paraformaldehyde for $20 \mathrm{~min}$ at room temperature. Cells were counterstained with DAPI to visualize cell nuclei and analyzed using an inverted fluorescence microscope (Olympus IX51) equipped with an Olympus Qcolor 3 digital camera (Olympus).

\section{Heat treatment}

TAECs were seeded onto the 6 -cm dishes, and after 24-h incubation they were exposed to heat treatment. Heat treatment was carried out by sealing the tops of culture plates with parafilm, and submerging the plates in a water bath set to the desired temperature for 10 min. We selected heat treatments of 42 and $47^{\circ} \mathrm{C}$ for 10 min to simulate the effects of insufficient RFA and $37^{\circ} \mathrm{C}$ for $10 \mathrm{~min}$ as the control treatment in vitro. Cell morphological changes were observed using an inverted fluorescence microscope (Olympus IX51) equipped with an Olympus Qcolor 3 digital camera (Olympus).

\section{TAEC proliferation, migration and tube formation after} heat treatment

TAEC proliferation was measured using a Cell Counting Kit-8 (CCK-8; Dojindo, Kumamoto, Japan) according to the manufacturer's instructions. Briefly, TAECs were cultured in 96-well plates at a concentration of $3 \times 10^{3} /$ well. After 24-h incubation, the plates were heat treated for $10 \mathrm{~min}$ at 37,42 or $47^{\circ} \mathrm{C}$. After incubation for 24,48 or $72 \mathrm{~h}, 5 \mu \mathrm{L}$ of CCK-8 reagent was added to each well. The absorbance was measured at $450 \mathrm{~nm}$ after 2.5 - $\mathrm{h}$ incubation at $37^{\circ} \mathrm{C}$.

TAECs were plated into the 6-well plates, and after 24-h incubation the plates were sealed and submerged for $10 \mathrm{~min}$ in a water bath set to 37,42 or $47^{\circ} \mathrm{C}$. At 24 , 48 or $72 \mathrm{~h}$ after heat treatment, TAECs were trypsinized and resuspended for further experiments involving migration and tube formation.

Quantitative cell migration assays were performed using a modified Boyden chamber (Costar-Corning, New York, USA) with 8.0- $\mu \mathrm{m}$ pore polycarbonate filter inserts in 24-well plates as described previously [9]. Briefly, the lower chamber was filled with EGM-2 with 10\% FBS, and TAECs $\left(5 \times 10^{4}\right.$ cells/well $)$ in serum-free medium were added into the upper chamber. The cells were allowed to migrate for $5 \mathrm{~h}$ at $37^{\circ} \mathrm{C}$. The non-migrated cells were removed from the upper surface of the membrane by scraping with a cotton swab, and the migrating cells were fixed with methanol, stained with crystal violet (Beyotime, Nantong, China) and photographed under an inverted fluorescence microscope (Olympus IX51) equipped with an Olympus Qcolor 3 digital camera (Olympus). Migration was assessed by counting the number of stained cells from 10 random fields at $\times 100$ magnification.

TAECs tube formation was studied on growth factorreduced Matrigel (Becton Dickinson, San Jose, USA) diluted 1:1 in ice with cold EBM-2 in a 96-well plate. Cells $\left(1 \times 10^{4}\right.$ cells/well $)$ were added to Matrigel-coated 96-well plates. TAECs were photographed under an inverted fluorescence microscope (Olympus IX51) equipped with an Olympus Qcolor 3 digital camera (Olympus). Tube formation was assessed by counting the number of loops from 10 random fields at $\times 50$ magnification.

\section{Collection of the conditioned medium and cell protein}

TAECs were cultured in a T25 culture bottle at a concentration of $5 \times 10^{5}$ cells in EGM-2 containing $10 \%$ FBS. After 24-h incubation, $1 \mathrm{ml}$ of fresh EBM-2 was added to the culture bottle with replacement of EGM-2. The culture bottles were submerged in a water bath for $10 \mathrm{~min}$ at 37,42 or $47^{\circ} \mathrm{C}$. Immediately after heat treatment, $2 \mathrm{ml}$ of fresh EBM-2 was added to each culture bottle and cells were cultured in the incubator at $37^{\circ} \mathrm{C}$. After 24-h incubation, the medium was collected and spun down at $3000 \mathrm{rpm}$ for $20 \mathrm{~min}$, and the supernatant was collected and stored at $-80^{\circ} \mathrm{C}$. Cells were lysed using cell lysis buffer $(150 \mathrm{mM} \mathrm{NaCl} ; 50 \mathrm{mM}$ Tris-HCl; pH 8.0; $0.1 \%$ SDS; and $1 \%$ Triton $\mathrm{X}-100)$ containing 
protease, phosphatase inhibitors, and protein was used for further experiments.

\section{Matrigel invasion assay}

The invasiveness of hepatoma cells was assessed by measuring their ability to invade through Matrigel-coated transwell inserts with 8.0- $\mu \mathrm{m}$ pores (Costar-Corning, New York, USA). HepG2-GFP or HCCLM3-GFP cells were trypsinized and resuspended with conditioned medium. $200 \mu \mathrm{l}$ of cell suspension $\left(2 \times 10^{4}\right.$ or $5 \times 10^{4}$ cells $)$ was added to each upper well, and the lower chamber was filled with DMEM with 10\% FBS. Hepatoma cells were allowed to invade for $36 \mathrm{~h}$ at $37^{\circ} \mathrm{C}$. The non-invaded cells were removed from the upper surface of the membrane by scraping with a cotton swab, and the invaded cells were fixed with methanol, stained with crystal violet, and photographed under inverted fluorescence microscopy (Olympus IX51) equipped with an Olympus Qcolor 3 digital camera (Olympus). Invasion was assessed by counting the number of stained cells from 10 random fields at $\times 100$ magnification.

\section{Enzyme-linked immunosorbent assay}

Cytokines secreted into the conditioned medium were quantified using an enzyme-linked immunosorbent assay (ELISA) kits (for IL-6, MCP-1, IL-8 and GRO- $\alpha$; Boster, Wuhan, China) according to the manufacturer's instructions. The concentration of cytokines was normalized to the total cellular protein.

\section{Western blot}

Equivalent amounts of whole cell extracts were subjected to SDS-PAGE gel and transferred to nitrocellulose membranes. The membranes were blocked with 5\% non-fat milk for $2 \mathrm{~h}$ and then incubated with respective primary antibody overnight at $4^{\circ} \mathrm{C}$ followed by the incubation with the appropriate HRP-conjugated secondary antibody for $1.5 \mathrm{~h}$ at room temperature. Blots were visualized with an ECL detection kit (Pierce, USA) and analyzed using Quantity One 1-D Analysis Software (Bio-Rad, Hercules, USA).

\section{TAEC-tumor cell adhesion assay}

TAECs were plated onto 96-well plates and allowed to grow until complete confluence was achieved. The plates were submerged in a water bath for $10 \mathrm{~min}$ at 37,42 or $47^{\circ} \mathrm{C}$. After $24-, 48-$ or $72-\mathrm{h}$ incubation, cells were rinsed with PBS twice, and HepG2-GFP or HCCLM3-GFP cells $\left(1 \times 10^{4}\right.$ cells $)$ in fresh DMEM were added to the wells. The plates were incubated at $37^{\circ} \mathrm{C}$ in $5 \% \mathrm{CO}_{2}$ for 20 $\mathrm{min}$. Non attached cells were removed by washing thrice with PBS and the attached hepatoma cells in each well were visualized under a microscope and counted using an inverted fluorescence microscope (Olympus IX51) equipped with an Olympus Qcolor 3 digital camera (Olympus).

\section{Cell surface adhesion molecules assay using cell ELISA}

TAECs were plated onto 96-well plates and allowed to grow until $80 \%$ confluence. The plates were submerged in a water bath for $10 \mathrm{~min}$ at 37,42 or $47^{\circ} \mathrm{C}$. After 24-, 48- or 72-h incubation, cells were washed with PBS twice and fixed with PBS containing 4\% paraformaldehyde at room temperature. The plates were blocked with $2 \% \mathrm{BSA}$ at $37^{\circ} \mathrm{C}$ for $2 \mathrm{~h}$. Cell surface expressions of adhesion molecules were determined by means of primary binding with specific antibody for VCAM-1, ICAM-1 or E-selectin (Santa Cruz, California, USA), followed by secondary binding with an HRP-conjugated goat antirabbit IgG antibody. Quantification was performed by determination of colorimetric conversion at OD at 450 $\mathrm{nm}$ of 3,3,5,5'-tetramethylbenzidine using a TMB peroxidase EIA substrate kit (Bio-Rad, Hercules, USA).

\section{Statistical analysis}

Student's $\mathrm{t}$ test or the ANOVA test was used for comparison of two groups or three groups using GraphPad Prism (GraphPad Software Inc., La Jolla, CA). A P value of $<0.05$ was set as the level of statistical significance.

\section{Results}

Isolation and characterization of TAECs from tumor tissue Seven strains of CD31+ TAECs were obtained from seven $\mathrm{HCC}$ patients. In addition to CD31, TAECs virtually expressed the EC-specific markers vWF, VEGFR1, VEGFR2 and CD34 (Figure 1A). Negative expression of CD68 and $\alpha$-SMA in isolated TAECs excluded the contamination of macrophages and fibroblasts (Figure 1A). Western blot confirmed the immunofluorescence results and that AFP expression could be detected in tumor cells but not in TAECs (Figure 1B). TAECs also took up complexes of Dil-Ac-LDL (Figure 1C).

\section{The effect of heat treatment on TAECs}

In order to simulate the growth pattern of TAECs that were not killed after the heat stress generated by RFA, TAECs were exposed to a 10-min heat treatment at temperatures ranging from 37 to $55^{\circ} \mathrm{C}$, and after $36 \mathrm{~h}$, morphological changes in TAECs were observed. It was found that TAECs could not be continuously cultured once the temperature exceeded $47^{\circ} \mathrm{C}$ (Figure 2A). To monitor the potential effect of insufficient RFA on the function of TAECs in vitro, we initially observed the cell proliferation, migration and tube formation of TAECs at 24, 48 and $72 \mathrm{~h}$ after heat treatment. It was found that the proliferation of TAECs after insufficient RFA was significantly decreased as compared 


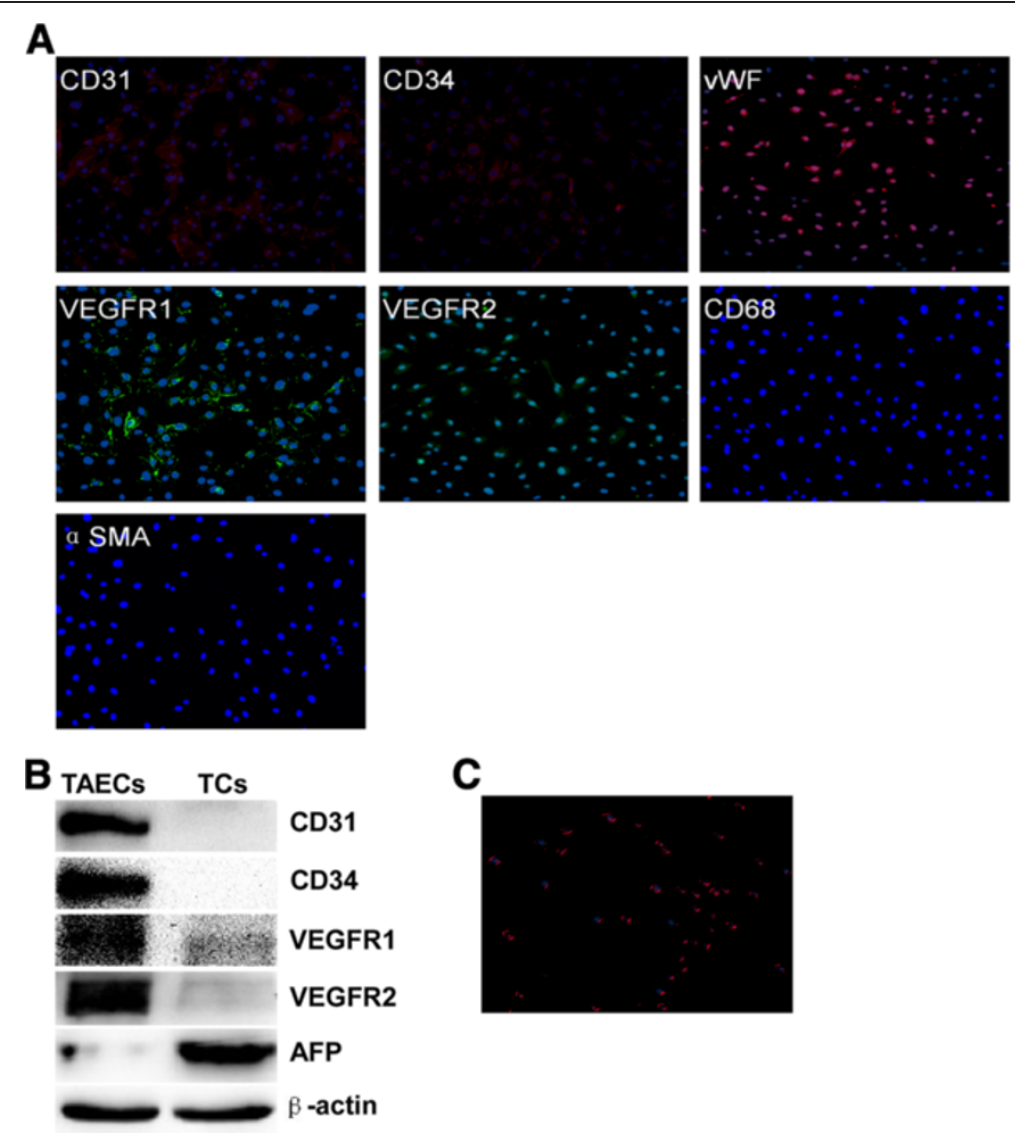

Figure 1 Verification of TAECs from HCC. (A) Representative immunofluorescence analysis of TAECs showing positive expression of the endothelial markers CD31, CD34, VEGFR1, VEGFR2 and VWF, and negative expression of the macrophages and fibroblast markers CD68 and a-SMA. (B) Western blot analysis used to verify the immunofluorescence results and assess the purity of the isolated CD31+ TAECs. (C) Micrograph representative of the uptake of Dil-Ac-LDL in TAECs. Five TAECs preparations were analyzed with similar results.

with the control treatment (Figure 2B). However, it was interesting that the insufficient RFA treatment promoted the migration and tube formation of TAECs; this was not observed after the control treatment (Figure 2C and D).

\section{Promotion of adhesion of TAECs to hepatoma cells after insufficient RFA}

HepG2-GFP or HCCLM3-GFP cells were used to investigate the adhesion ability of TAECs after insufficient RFA. TAECs were treated with insufficient RFA or the control treatment. After a 24-, 48- or 72-h interval, they were incubated at $37^{\circ} \mathrm{C}$ and hepatoma cells were added. The results showed that after insufficient RFA TACEs adhered to HepG2-GFP cells at a significantly higher level than the case after the control treatment (all $P<0.001$; Figure $3 \mathrm{~A}$ and $\mathrm{B}$ ). Similar results were observed in HCCLM3-GFP cells (all $P<0.001$; Additional file 1: Figure S1). In order to explore the mechanism involved in the process, we measured the surface expressions of the TAEC adhesion molecules after insufficient RFA using cell ELISA. The results showed that the expression of E-selectin, intercellular adhesion molecule (ICAM)-1 and vascular cell adhesion molecule (VCAM)-1 were significantly up-regulated on the surface of TAECs at 24, 48 and $72 \mathrm{~h}$ after insufficient RFA (Figure 3B). Western blot also confirmed the cell ELISA results (Figure $3 \mathrm{C}$ ).

\section{Promotion of the invasiveness of hepatoma cells by TAECs after insufficient RFA}

Using the conditioned media from TAECs with or without insufficient RFA treatment, we further explored the effect of TAECs on the invasiveness of hepatoma cells. Conditioned medium from TAECs after insufficient RFA significantly enhanced the invasiveness of HepG2-GFP cells relative to the control (Figure 4A). Similar results were observed in HCCLM3-GFP cells (Additional file 2: Figure S2). To test the possible mechanism involved in the promotion of the invasiveness 
A
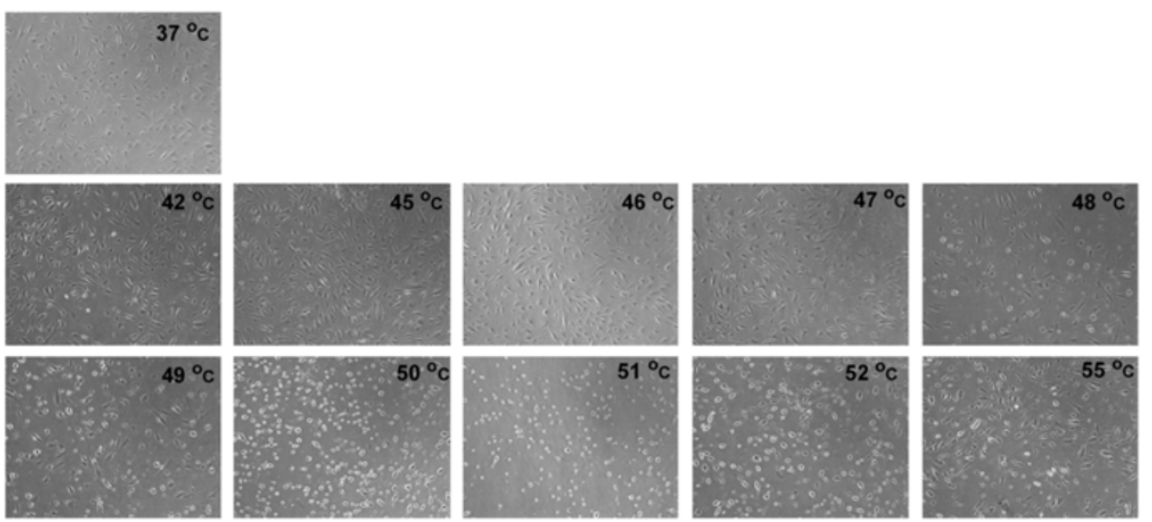

B
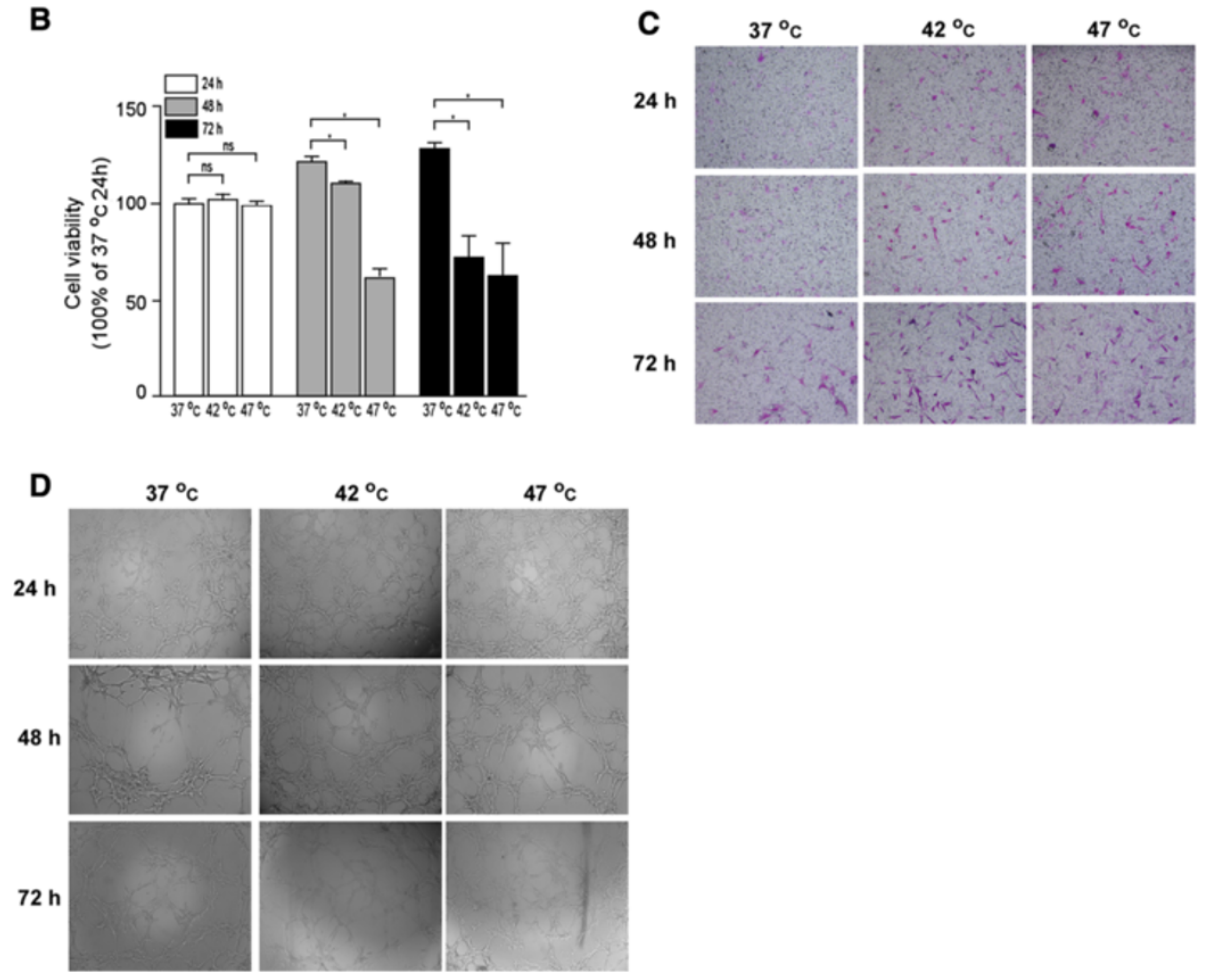

Figure 2 Morphological changes in TAECs after heat treatment at different temperatures and inhibition of their proliferation and enhancement of their migration and tube formation after insufficient RFA. (A) Representative micrograph of morphological change in TAECs after a 10-min heat treatment at temperatures ranging from 37 to $55^{\circ} \mathrm{C}$. (B) TAECs were cultured after insufficient RFA $\left(42\right.$ or $47^{\circ} \mathrm{C}$ treatment for $10 \mathrm{~min}$ ). The proliferation of TAECs at 24,48 and $72 \mathrm{~h}$ after heat treatment were measured using the CCK- 8 assay. Columns: means from three individual experiments with five samples per group; bars: SE; ${ }^{*}: P<0.05$; ns: no significance. (C) TAECs were cultured after insufficient RFA and the migration of TAECs at 24, 48 and $72 \mathrm{~h}$ were measured using the trans-well assay. Representative micrographs of migration are shown. (D) TAECs were cultured after insufficient RFA and TAEC tube formation at 24, 48 and $72 \mathrm{~h}$ was evaluated using the Matrigel tube formation assay. Representative micrographs of tube formation are displayed. Data represent the representative results from three independent experiments with five samples per group.

of hepatoma cells by TAECs after insufficient RFA, we measured the levels of cytokine secreted by TAECs in the conditioned medium. We found that insufficient RFA significantly increased the secreted levels of IL-8, IL-6, MCP- 1 and GRO- $\alpha$ by TAECs (all $P<0.05$; Figure $4 \mathrm{~B})$.
Enhancement of the activity of ERK1/2, NF-KB and Akt signaling pathways and inhibition of STAT3 signaling pathway after insufficient RFA

To further determined the associated signal pathways involved in the process as described above, we investigated the expression levels of total and phosphorylated 

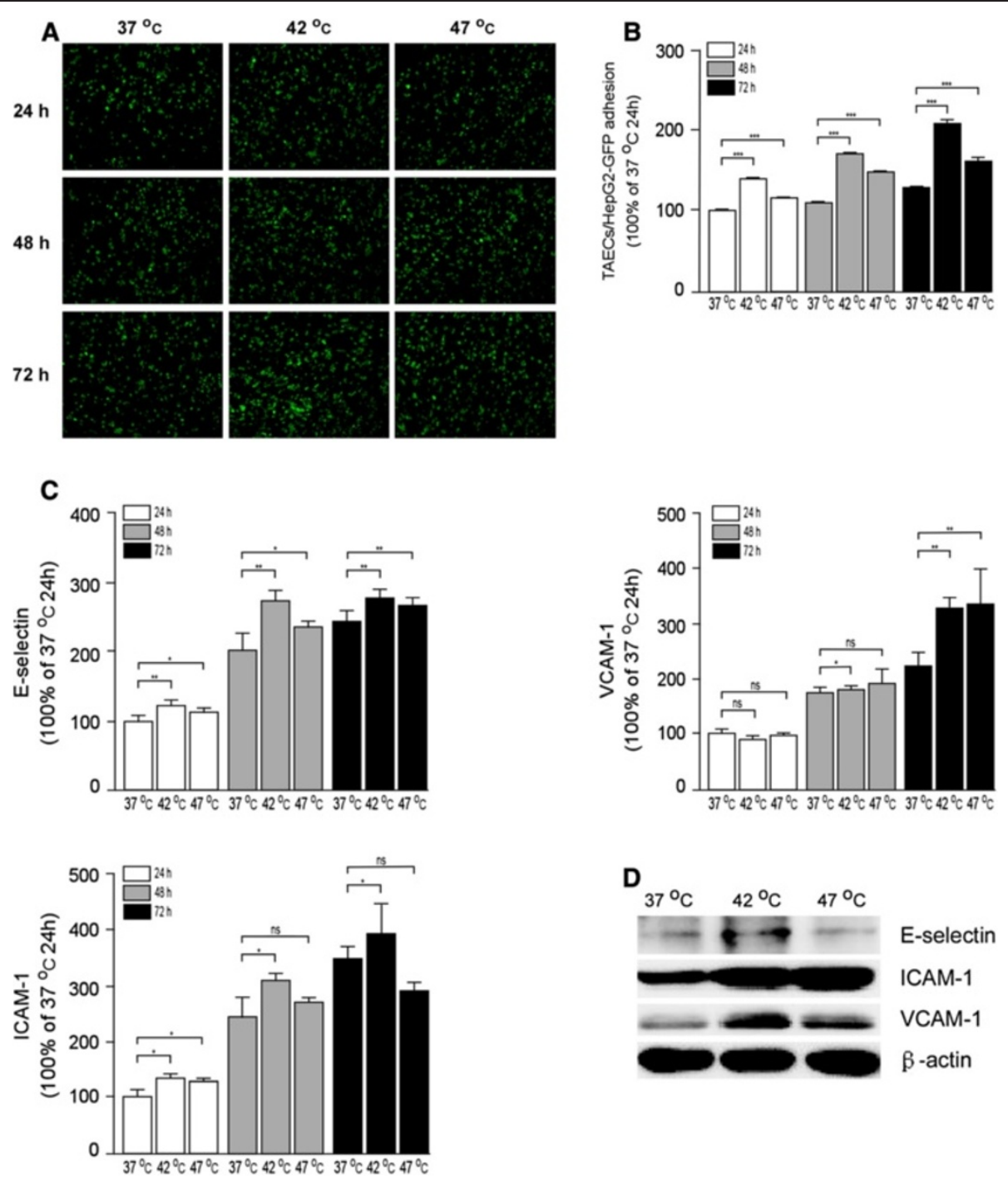

Figure 3 Increased TAEC interaction with tumor cells and up-regulated expression of E-selectin, ICAM-1 and VCAM-1 after insufficient RFA. (A-B) TAECs were cultured after insufficient RFA, and HepG2-GFP cells were added after 24, 48 and $72 \mathrm{~h}$. Representative micrographs of TAECs regarding the interaction with HepG2-GFP cells are shown. Columns: means from three individual experiments with five samples per group; bars: SE; **: $P<0.001$. (C) The expression of E-selectin, ICAM-1 and VCAM-1 on the surface of TAECs after insufficient RFA was detected using cell ELISA analysis. Columns: means from three individual experiments with five samples per group; bars: $\mathrm{SE} ;{ }^{*}$ : $P<0.05 ;{ }^{*}$ : $P<0.01$; ns: no significance. (D) Expression of E-selectin, ICAM-1 and VCAM-1 in TAECs after insufficient RFA detected by western blot analysis. Data are the representative results of three independent experiments with five samples per group.

ERK1/2, NF-kB, Akt and STAT3 protein in TAECs at 24 $h$ after insufficient RFA. It was found that total protein levels of ERK1/2, NF-кB, Akt and STAT3 were not changed after insufficient RFA, whereas phosphorylated ERK1/2 (p-ERK1/2), NF-kB and p-Akt were upregulated and $\mathrm{p}$-STAT3 was substantially downregulated in TAECs after insufficient RFA (Figure 5).

\section{Discussion}

RFA heats tumor tissue owing to ionic friction generated by the radiofrequency current, which induces coagulation necrosis once the tissue temperature exceeds $50^{\circ} \mathrm{C}$ for 4-6 min [23]. If the HCC tumor is not completely coagulated, the residual tumor cells are prone to proliferation, invasion and angiogenesis [9-11]. On the other hand non-tumor cells, especially TAECs, are also exposed to RFA, and insufficient RFA can theoretically influence the behavior of these cells. It remains poorly understood as to whether or not TAECs promote the metastasis of hepatoma cells after insufficient RFA.

The growth and migration of endothelial cells are essential for tumor angiogenesis [24]. In the absence of local neovascular formation, the tumor may not grow beyond $2-3 \mathrm{~mm}$ in diameter [25]. Most of the previous 
A
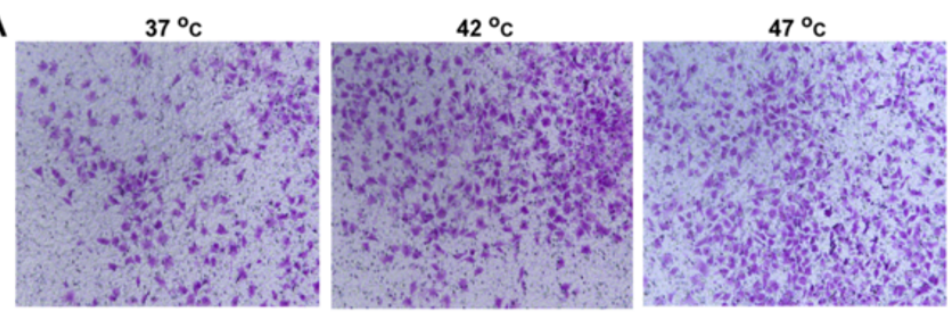

B
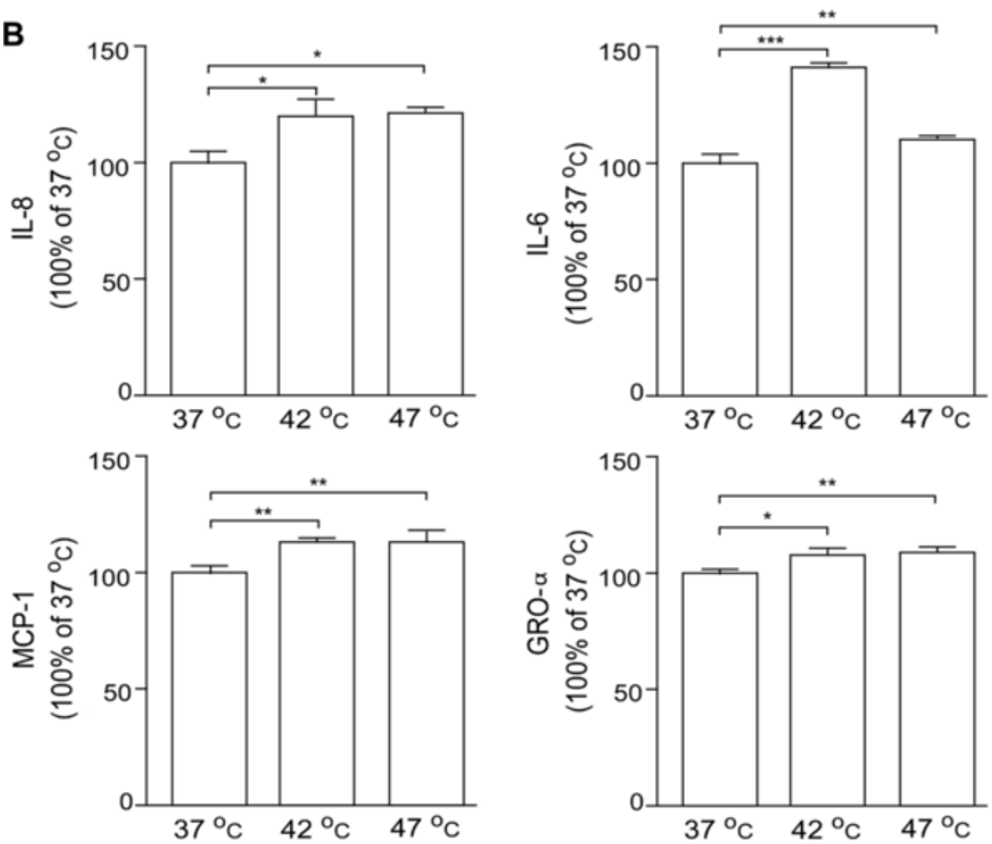

Figure 4 Promotion of the invasiveness of hepatoma cells by TAECs after insufficient RFA. (A) HepG2-GFP invasion in vitro in response to conditioned media from TAECs was assayed after the control treatment or insufficient RFA. Data are the representative results of three independent experiments with five samples per group. (B) IL-6, IL-8, MCP-1 and GRO-a concentrations in conditioned medium were detected using the ELISA assay. Columns: means from three individual experiments with five samples per group; bars: $\mathrm{SE}^{*}{ }^{*}: P<0.05$; ${ }^{* *}: P<0.01$; ${ }^{* * *}$ : $P<0.001$.

studies on tumor angiogenesis have been conducted using normal endothelial cells (NECs) such as human umbilical vein endothelial cells. The use of NECs does not reflect the real tumor microenvironment. In contrast TAECs, which express tumor-specific endothelial markers, are cytogenetically abnormal and genetically unstable [18,19]. TAECs also manifest an increased angiogenesis capability and drug resistance, and display resistance to interferon $\gamma$ as compared with NECs under hypoxia $[22,26]$. Accordingly, the use of TAECs as a tool to research angiogenesis virtually reflects the microenvironment of tumor. Here, for the first time, we have observed the effect of insufficient RFA on TAECs in vitro. We isolated the CD31+ TAECs from fresh HCC tissue and found that insufficient RFA enhanced the migration and tube formation of these cells, but inhibited their proliferation. The results revealed that after insufficient RFA TAECs may enhance autochthonous angiogenesis to promote the rapid growth of residual HCC.
The reason that the proliferation of TAECs was inhibited may be that insufficient RFA induced cell apoptosis and/ or mitotic failure over a short time period.

Metastasis results from completion of a complex succession of cell-biological events-collectively termed the invasion-metastasis cascade. The process of cancer cell metastasis consists of: (1) local invasion through the surrounding extracellular matrix (ECM) and stromal cell layers; (2) intravasation into the lumina of blood vessels; (3) survival of the rigors of transport through the vasculature; (4) arrest at distant organ sites; (5) extravasation into the parenchyma of distant tissues; (6) initial survival in these foreign microenvironments in order to form micrometastases; and (7) reinitiation of proliferative programs at metastatic sites, thereby generating macroscopic, clinically detectable neoplastic growths [27]. Each of these processes involves rate-limiting steps that are influenced by non-malignant cells in the tumor microenvironment [12]. Previous studies have also 


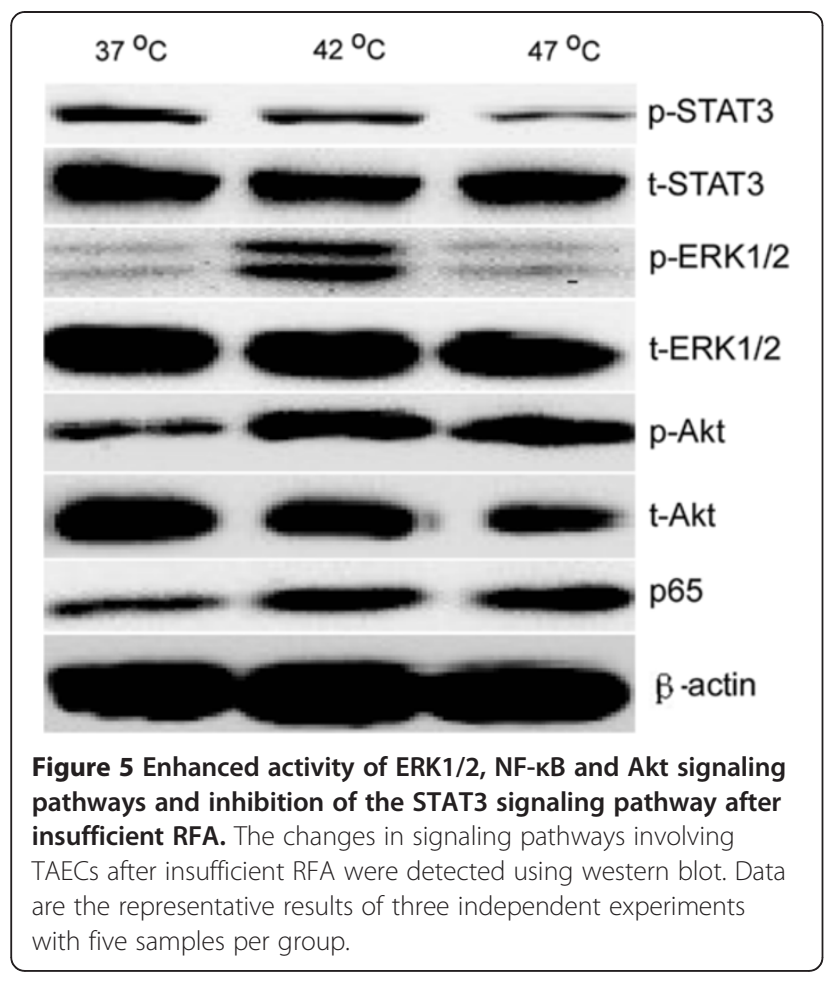

shown that the altered microenvironment promotes the outgrowth and metastasis of residual tumor cells. However, it has not previously been reported that a nonmalignant cell type in vitro can contribute adversely to the altered microenvironment after insufficient RFA.

It is known that the first step in metastasis is local invasion of hepatoma cells through the surrounding ECM. In present study, we found that TAECs significantly promoted hepatoma cells cell invasion through Matrigel in vitro after insufficient RFA. This suggested that cytokines secreted by TAECs in conditioned medium after insufficient RFA led to the increased invasiveness of the hepatoma cells. TAECs can secrete many cytokines, for example IL-8, IL-6, MCP-1 and GRO- $\alpha$ [17]. IL-8 plays an important role in inflammation, tumor-induced angiogenesis and tumor metastasis and IL-6 is involved in the proliferation, differentiation and metastasis of various malignant tumor cells [28-30]. MCP-1 secreted by hepatic myofibroblasts promotes the migration and invasion of human hepatoma cells, and MCP-1 suppresses the inhibition of tumor growth and metastasis in lung cancer and HCC [31-33]. High serum levels of IL6, IL-8 and MCP-1 have been shown to be positively correlated with tumor development in cancer patients [34-36]. GRO- $\alpha$ plays an important role with regard to disease progression and metastasis formation and has also been demonstrated to be positively associated with tumor size, stage, invasion, lymph node metastasis and patient survival in colorectal cancers $[37,38]$. In the present study, we discovered that cytokines secreted by TAECs including IL-6, IL-8, MCP-1 and GRO- $\alpha$ were up-regulated after insufficient RFA, which may explain the enhanced invasiveness ability of hepatoma cells.

The second step in metastasis involves the intravasation of hepatoma cells into the lumens of blood vessels, and the interaction of hepatoma cells with TAECs plays an important role in the process. In our study we observed that after insufficient RFA TACEs adhered to significantly more hepatoma cells. Meanwhile E-selectin, ICAM-1 and VCAM-1, which are all expressed on vascular endothelial cells, have been found to be responsible for the formation of firm adhesion between the tumor and the endothelium [39-42]. Our results revealed that insufficient RFA up-regulated the expression of E-selectin, ICAM-1 and VCAM-1 in TAECs, which suggests that up-regulated adhesion molecules may be the mechanism responsible for the enhanced adhesion of TAECs to hepatoma cells.

The biological changes in TAECs after insufficient RFA must involve various cell signal pathways. Activation of Akt, NF-kB, STAT3 and ERK1/2 was found in HCC, and this was associated with tumor cell survival, proliferation, invasiveness and metastasis [43-46]. In our previous study we also found that insufficient RFA activated the p-Akt/HIF-1 $\alpha$ /VEGFA signal pathway of hepatoma cells promoting angiogenesis in residual HCC [9]. Our present study revealed that insufficient RFA could up-regulate the expression of p-ERK1/2, p-Akt and NF$\kappa B$ and down-regulate the expression of p-STAT3 in TAECs. The up-regulated expression of $\mathrm{p}$-ERK1/2, $\mathrm{p}$ Akt and NF- $\mathrm{KB}$ may explain the phenomenon as described above. However, in the present study, we could not identify which signal pathway played a key role in the promotion of rapid growth and metastasis in residual HCC by TAECs, and why the expression of pSTAT3 in TAECs was down-regulated after insufficient RFA. Further study is needed in the future to clarify the exact mechanism involved in the signal pathway associated with the biological behavior of TAECs after insufficient RFA.

Combined with the findings from our previous research, our present results suggest that insufficient RFA affects not only hepatoma cells but also TAECs within the HCC. Such effects should be taken into account in the treatment of HCC using RFA. Anti-angiogenesis drugs, which are used to target TAECs, may be useful in preventing the rapid growth and metastasis of residual HCC after insufficient RFA.

\section{Conclusions}

Enhanced TAEC migration and tube formation after insufficient RFA may play a key role in the rapid growth of residual HCC. Increased expression of metastasis-related 
molecules in TAECs after insufficient RFA may be a possible mechanism for the metastasis of residual HCC.

\section{Additional files}

Additional file 1: Figure S1. Increased TAEC interaction with HCCLM3GFP cells after insufficient RFA. (A-B) TAECs were cultured after insufficient RFA, and HCCLM3-GFP cells were added after 24, 48 and $72 \mathrm{~h}$. Representative micrographs of TAECs regarding the interaction with HCCLM3-GFP cells are shown. Columns: means from three individual experiments with five samples per group; bars: SE; ${ }^{* * *}$ : $P<0.001$.

Additional file 2: Figure S2. Promotion of the invasiveness of HCCLM3GFP cells by TAECs after insufficient RFA. HCCLM3-GFP invasion in vitro in response to conditioned media from TAECs was assayed after the control treatment or insufficient RFA. Representative micrographs of HCCLM3GFP cell invasion are shown. Data are the representative results of three independent experiments with five samples per group.

\section{Competing interests}

The authors declare that they have no competing interests.

\section{Authors' contributions}

JK carried out the molecular biology studies, participated in the sequence alignment and drafted the manuscript. LQK and SK carried out the immunoassays. JGK, JG, LMZ and XMD participated in the sequence alignment. HCS participated in the design of the study and performed the statistical analysis. WBS conceived of the study, and participated in its design and coordination and helped to draft the manuscript. All authors read and approved the final manuscript.

\section{Acknowledgements}

This project was supported by the National Natural Science Foundation of China (grant number: 81172320) and the Dr Wu Jie-ping Medical Foundation (grant number: 320.6750.07131).

\section{Author details}

${ }^{1}$ Department of Hepatobiliary Surgery, Beijing Chaoyang Hospital, Capital Medical University, Beijing, China. 'Liver Cancer Institute, Zhongshan Hospital, Fudan University, Shanghai, China. ${ }^{3}$ The Institute of Cardiovascular Sciences and Institute of Systems Biomedicine, School of Basic Medical Sciences, Peking University Health Science Center, Key Laboratory of Molecular Cardiovascular Sciences of Education Ministry, and Key Laboratory of Cardiovascular Molecular Biology and Regulatory Peptides of Health Ministry, Beijing, China.

Received: 16 July 2012 Accepted: 1 November 2012

Published: 21 November 2012

\section{References}

1. Forner A, Llovet JM, Bruix J: Hepatocellular carcinoma. Lancet 2012, 379:1245-1255.

2. Nathan H, Bridges JF, Schulick RD, Cameron AM, Hirose K, Edil BH, Wolfgang $\mathrm{CL}$, Segev DL, Choti MA, Pawlik TM: Understanding surgical decision making in early hepatocellular carcinoma. J Clin Oncol 2011, 29:619-625.

3. Rossi S, Ravetta V, Rosa L, Ghittoni G, Viera FT, Garbagnati F, Silini EM, Dionigi P, Calliada F, Quaretti P, Tinelli C: Repeated radiofrequency ablation for management of patients with cirrhosis with small hepatocellular carcinomas: a long-term cohort study. Hepatology 2011, 53:136-147.

4. Nijkamp MW, van der Bilt JD, de Bruijn MT, Molenaar IQ, Voest EE, van Diest PJ, Kranenburg O, Borel Rinkes IH: Accelerated perinecrotic outgrowth of colorectal liver metastases following radiofrequency ablation is a hypoxia-driven phenomenon. Ann Surg 2009, 249:814-823.

5. Feng K, Yan J, Li X, Xia F, Ma K, Wang S, Bie P, Dong J: A randomized controlled trial of radiofrequency ablation and surgical resection in the treatment of small hepatocellular carcinoma. J Hepatol 2012, 57:794-802.

6. Koda M, Murawaki Y, Hirooka Y, Kitamoto M, Ono M, Sakaeda H, Joko K, Sato S, Tamaki K, Yamasaki T, Shibata H, Shimoe T, Matsuda T, Toshikuni N, Fujioka SI, Ohmoto K, Nakamura S, Kariyama K, Aikata H, Kobayashi Y,
Tsutsui A: Complications of radiofrequency ablation for hepatocellular carcinoma in a multicenter study: An analysis of 16346 treated nodules in 13283 patients. Hepatol Res 2012, 42:1058-1064.

7. Kasugai H, Osaki Y, Oka H, Kudo M, Seki T: Severe complications of radiofrequency ablation therapy for hepatocellular carcinoma: an analysis of 3,891 ablations in 2,614 patients. Oncology 2007, 72(Suppl 1):72-75.

8. Ruzzenente A, Manzoni GD, Molfetta M, Pachera S, Genco B, Donataccio M, Guglielmi A: Rapid progression of hepatocellular carcinoma after Radiofrequency Ablation. World J Gastroenterol 2004, 10:1137-1140.

9. Kong J, Pan B, Ke S, Dong S, Li X, Zhou A, Zheng L, Sun WB: Insufficient Radiofrequency Ablation Promotes Angiogenesis of Residual Hepatocellular Carcinoma via HIF-1alpha/VEGFA. PLoS One 2012, 7:e37266.

10. Ke S, Ding XM, Kong J, Gao J, Wang SH, Cheng Y, Sun WB: Low temperature of radiofrequency ablation at the target sites can facilitate rapid progression of residual hepatic VX2 carcinoma. J Trans/ Med 2010, 8:73.

11. Obara K, Matsumoto N, Okamoto M, Kobayashi M, Ikeda H, Takahashi H, Katakura Y, Matsunaga K, Ishii T, Okuse C, Suzuki M, Itoh F: Insufficient radiofrequency ablation therapy may induce further malignant transformation of hepatocellular carcinoma. Hepatol Int 2008, 2:116-123.

12. Joyce JA, Pollard JW: Microenvironmental regulation of metastasis. Nat Rev Cancer 2009, 9:239-252.

13. von Breitenbuch $P$, Kohl G, Guba M, Geissler E, Jauch KW, Steinbauer M: Thermoablation of colorectal liver metastases promotes proliferation of residual intrahepatic neoplastic cells. Surgery 2005, 138:882-887.

14. Nikfarjam M, Muralidharan V, Christophi C: Altered growth patterns of colorectal liver metastases after thermal ablation. Surgery 2006, 139:73-81.

15. Nijkamp MW, Hoogwater FJ, Steller EJ, Westendorp BF, van der Meulen TA, Leenders MW, Borel Rinkes $\mathrm{H}$, Kranenburg O: CD95 is a key mediator of invasion and accelerated outgrowth of mouse colorectal liver metastases following radiofrequency ablation. J Hepatol 2010, 53:1069-1077.

16. Bussolati B, Deambrosis I, Russo S, Deregibus MC, Camussi G: Altered angiogenesis and survival in human tumor-derived endothelial cells. FASEB J 2003, 17:1159-1161.

17. Knipe L, Meli A, Hewlett L, Bierings R, Dempster J, Skehel P, Hannah MJ, Carter T: A revised model for the secretion of tPA and cytokines from cultured endothelial cells. Blood 2010, 116:2183-2191.

18. Akino T, Hida K, Hida Y, Tsuchiya K, Freedman D, Muraki C, Ohga N, Matsuda K, Akiyama K, Harabayashi T, Shinohara N, Nonomura K, Klagsbrun M, Shindoh M: Cytogenetic abnormalities of tumor-associated endothelial cells in human malignant tumors. Am J Pathol 2009, 175:2657-2667.

19. Hida K, Hida Y, Amin DN, Flint AF, Panigrahy D, Morton CC, Klagsbrun M: Tumor-associated endothelial cells with cytogenetic abnormalities. Cancer Res 2004, 64:8249-8255.

20. Liang Y, Li WW, Yang BW, Tao ZH, Sun HC, Wang L, Xia JL, Qin LX, Tang ZY, Fan J, Wu WZ: Aryl hydrocarbon receptor nuclear translocator is associated with tumor growth and progression of hepatocellular carcinoma. Int J Cancer 2012, 130:1745-1754.

21. Yang BW, Liang $Y$, Xia JL, Sun HC, Wang L, Zhang JB, Tang ZY, Liu KD, Chen J, Xue Q, Gao DM, Wu WZ: Biological characteristics of fluorescent protein-expressing human hepatocellular carcinoma xenograft model in nude mice. Eur J Gastroenterol Hepatol 2008, 20:1077-1084.

22. Xiong $Y Q$, Sun $H C$, Zhang W, Zhu XD, Zhuang PY, Zhang JB, Wang L, W WZ, Qin LX, Tang ZY: Human hepatocellular carcinoma tumor-derived endothelial cells manifest increased angiogenesis capability and drug resistance compared with normal endothelial cells. Clin Cancer Res 2009, 15:4838-4846.

23. Lencioni R: Loco-regional treatment of hepatocellular carcinoma. Hepatology 2010, 52:762-773.

24. Lamalice L, Le Boeuf F, Huot J: Endothelial cell migration during angiogenesis. Circ Res 2007, 100:782-794.

25. Folkman J: Angiogenesis in cancer, vascular, rheumatoid and other disease. Nat Med 1995, 1:27-31.

26. Wang JH, Wu QD, Bouchier-Hayes D, Redmond HP: Hypoxia upregulates $\mathrm{Bcl}-2$ expression and suppresses interferon-gamma induced antiangiogenic activity in human tumor derived endothelial cells. Cancer 2002, 94:2745-2755 
27. Valastyan S, Weinberg RA: Tumor metastasis: molecular insights and evolving paradigms. Cell 2011, 147:275-292.

28. Adachi Y, Yoshio-Hoshino N, Nishimoto N: The blockade of IL-6 signaling in rational drug design. Curr Pharm Des 2008, 14:1217-1224.

29. Guo Y, XU F, Lu T, Duan Z, Zhang Z: Interleukin-6 signaling pathway in targeted therapy for cancer. Cancer Treat Rev 2012:

30. Fitzgerald JP, Nayak B, Shanmugasundaram K, Friedrichs W, Sudarshan S, Eid AA, DeNapoli T, Parekh DJ, Gorin Y, Block K: Nox4 mediates renal cell carcinoma cell invasion through hypoxia-induced interleukin 6- and 8production. PLoS One 2012, 7:e30712.

31. Chen TA, Wang JL, Hung SW, Chu CL, Cheng YC, Liang SM: Recombinant VP1, an Akt inhibitor, suppresses progression of hepatocellular carcinoma by inducing apoptosis and modulation of CCL2 production. PLoS One 2011, 6:e23317.

32. Dagouassat M, Suffee $N$, Hlawaty H, Haddad O, Charni F, Laguillier C, Vassy R, Martin L, Schischmanoff PO, Gattegno L, Oudar O, Sutton A, Charnaux N Monocyte chemoattractant protein-1 (MCP-1)/CCL2 secreted by hepatic myofibroblasts promotes migration and invasion of human hepatoma cells. Int J Cancer 2010, 126:1095-1108.

33. Fridlender ZG, Kapoor V, Buchlis G, Cheng G, Sun J, Wang LC, Singhal S, Snyder LA, Albelda SM: Monocyte chemoattractant protein-1 blockade inhibits lung cancer tumor growth by altering macrophage phenotype and activating CD8+ cells. Am J Respir Cell Mol Biol 2011, 44:230-237.

34. Chen Z, Malhotra PS, Thomas GR, Ondrey FG, Duffey DC, Smith CW, Enamorado I, Yeh NT, Kroog GS, Rudy S, McCullagh L, Mousa S, Quezado M, Herscher $L L$, Van Waes $C$ : Expression of proinflammatory and proangiogenic cytokines in patients with head and neck cancer. Clin Cancer Res 1999, 5:1369-1379.

35. Ren Y, Poon RT, Tsui HT, Chen WH, Li Z, Lau C, Yu WC, Fan ST: Interleukin-8 serum levels in patients with hepatocellular carcinoma: correlations with clinicopathological features and prognosis. Clin Cancer Res 2003, 9:5996-6001.

36. Lebrecht A, Grimm C, Lantzsch T, Ludwig E, Hefler L, Ulbrich E, Koelbl H: Monocyte chemoattractant protein-1 serum levels in patients with breast cancer. Tumour Biol 2004, 25:14-17.

37. Strell C, Niggemann B, Voss MJ, Powe DG, Zanker KS, Entschladen F: Norepinephrine promotes the beta1-integrin-mediated adhesion of MDA-MB-231 cells to vascular endothelium by the induction of a GROalpha release. Mol Cancer Res 2012, 10:197-207.

38. Ogata H, Sekikawa A, Yamagishi H, Ichikawa K, Tomita S, Imura J, Ito Y, Fujita M, Tsubaki M, Kato H, Fujimori T, Fukui H: GROalpha promotes invasion of colorectal cancer cells. Oncol Rep 2010, 24:1479-1486.

39. Daneker GW, Lund SA, Caughman SW, Staley CA, Wood WC: Anti-metastatic prostacyclins inhibit the adhesion of colon carcinoma to endothelial cells by blocking E-selectin expression. Clin Exp Metastasis 1996, 14:230-238.

40. Brodt P, Fallavollita L, Bresalier RS, Meterissian S, Norton CR, Wolitzky BA: Liver endothelial E-selectin mediates carcinoma cell adhesion and promotes liver metastasis. Int J Cancer 1997, 71:612-619.

41. Neeson PJ, Thurlow PJ, Jamieson GP, Bradley C: Lymphocyte-facilitated tumour cell adhesion to endothelial cells: the role of high affinity leucocyte integrins. Pathology 2003, 35:50-55.

42. Yamada M, Yanaba K, Hasegawa M, Matsushita Y, Horikawa M, Komura K, Matsushita T, Kawasuji A, Fujita T, Takehara K, Steeber DA, Tedder TF, Sato S: Regulation of local and metastatic host-mediated anti-tumour mechanisms by L-selectin and intercellular adhesion molecule-1. Clin Exp Immunol 2006, 143:216-227.

43. Cully M, You H, Levine AJ, Mak TW: Beyond PTEN mutations: the PI3K pathway as an integrator of multiple inputs during tumorigenesis. Nat Rev Cancer 2006, 6:184-192.

44. He G, Karin M: NF-kappaB and STAT3 - key players in liver inflammation and cancer. Cell Res 2011, 21:159-168.

45. Yu H, Pardoll D, Jove R: STATs in cancer inflammation and immunity: a leading role for STAT3. Nat Rev Cancer 2009, 9:798-809.

46. Wang S, Huang X, Li Y, Lao H, Zhang Y, Dong H, Xu W, Li J, Li M: RN181 suppresses hepatocellular carcinoma growth by inhibition of the ERK/ MAPK pathway. Hepatology 2011, 53:1932-1942.

\section{Submit your next manuscript to BioMed Central and take full advantage of:}

- Convenient online submission

- Thorough peer review

- No space constraints or color figure charges

- Immediate publication on acceptance

- Inclusion in PubMed, CAS, Scopus and Google Scholar

- Research which is freely available for redistribution 